\title{
Editorial
}

Nephrology

\section{Immunosuppressive Treatment in C3 Glomerulopathy: Time to Reconsider Our Approach}

\author{
Vladimir Tesar Zdenka Hruskova \\ Department of Nephrology, 1st Faculty of Medicine, Charles University and General University Hospital, Prague, \\ Czech Republic
}

Caliskan et al. [1] in their retrospective analysis compared the outcomes of 66 patients with C3 glomerulopathy who were treated with mycophenolate mofetil, with cyclophosphamide-based treatment, or by adopting conservative treatment methods. The primary outcome (chronic kidney disease stage 5 or decrease of estimated glomerular filtration rate [eGFR] by more than $50 \%$ ) was not significantly different between the groups with $25.8 \%$ of patients achieving the primary outcome within the median follow-up of 28 months. Numerically the percentage of patients achieving the primary outcome was similar among mycophenolate-treated patients and patients on conservative treatment (29.6 vs. $31.3 \%$, respectively). Clinical predictors of poor outcome were young age, higher proteinuria, and lower eGFR at presentation. With regard to the histology, poor outcome was related to the percentage of crescentic and sclerotic glomeruli and to the severity of interstitial fibrosis.

C3 glomerulopathy, a newly defined subgroup of membranoproliferative immunoglobuline-negative glomerulonephritis (MPGN), includes dense deposit disease (DDD, formerly MPGN type 2) and immunoglobulinnegative type I and type III MPGN (C3 glomerulonephritis). Its pathogenesis is characterized by the dysregulation of the alternative complement pathway, and the terminal complement complex is entirely different compared to MPGN with immunoglobulin deposits [2]. The pathogenesis of $\mathrm{C} 3$ deposition in the glomeruli of immunoglobulin-negative MPGN is, however, very heterogenous and includes, in a minority of patients $(<20 \%)$, various genetic causes, for example, mutations of $\mathrm{CFH}, \mathrm{CFI}$, and in CFHR5 nephropathy, most frequently internal duplication of CFHR 5 gene. Additionally, the $\mathrm{C} 3$ glomerulopathy may be caused by the not routinely detected autoantibodies directed at complement factor $\mathrm{B}, \mathrm{CFH}$, and autoantibodies stabilizing C3 convertase - $\mathrm{C} 3 \mathrm{bBb}$, previously called "nephritic factor" (C3 NeF) [3]. Unfortunately, in clinical practice, etiology remains unknown in most patients.

The outcome of patients with C3 glomerulonephritis is poor with $>50 \%$ having substantial renal dysfunction within 12 years [4]. However, the outcome was initially predicted to be better than that of DDD, as $>50 \%$ of patients with DDD progress to end-stage renal disease (ESRD) within 10 years [5]. In a recent paper [6], patients with DDD were compared to those with C3 glomerulonephritis, those who were younger and to those who tended to have lower serum C3 levels and greater probability of crescentic GN. Conversely, patients with C3 glomerulonephritis were older and had more severe arterio-

\section{KARGER}

(c) 2017 S. Karger AG, Basel

E-Mail karger@karger.com

www.karger.com/ajn
Vladimir Tesar

Department of Nephrology

1st Faculty of Medicine, Charles University and General University Hospital

U nemocnice 2, CZ-128 08 Prague 2 (Czech Republic)

E-Mail vladimir.tesar@vfn.cz 
lar sclerosis, glomerular sclerosis, and interstitial fibrosis. Age, DDD, and crescentic GN were identified as risk factors for ESRD progression (29\% of patients reached ESRD within a mean follow-up of 28 months). Cumulative renal survival was, however, not different in DDD, and C3 glomerulonephritis as progression was delayed only in patients with $\mathrm{C} 3 \mathrm{GN}$, and outcomes of $\mathrm{C} 3$ glomerulonephritis were poor with high risk of progression to ESRD [6].

Age, decreased eGFR, and percentage of crescents (not defined as cellular, or fibrous, or both and if segmental, or global) were also confirmed as risk factors of progression in the study of Caliskan et al. [1]. Moreover, this study also identified other risk factors for progression, namely, the percentage of sclerotic glomeruli and severity of interstitial fibrosis.

There are insufficient and controversial data about the treatment efficacy of C3 GN and no therapeutic standard is defined with which novel strategies aimed directly at complement activation could be compared. KDIGO Guidelines from 2012 about the treatment of glomerulonephritis suggest that patients with presumed idiopathic MPGN and nephrotic syndrome with progressive decline in kidney function be treated with either oral cyclophosphamide or mycophenolate mofetil (MMF) plus corticosteroids. As C3 glomerulopathy was defined only recently, it is difficult to use older data for treatment of MPGN to assess the treatment responsiveness of $\mathrm{C} 3$ glomerulopathy. Recent studies reported the ineffectiveness of corticosteroids and mycophenolate [7] in both DDD and C3 glomerulonephritis. Experience with plasmapheresis is limited and was also unsuccessful in DDD [7].

Recently, the largest retrospective analysis with a median follow-up of 47 months focused on the effectiveness of antiproliferative treatment in 60 Spanish patients with C3 glomerulonephritis [8]. The investigators compared corticosteroid with mycophenolate (22 patients), corticosteroids alone or combined with cyclophosphamide (18 patients) with no immunosuppressive treatment (20 patients). Mycophenolate mofetil-based treatment was significantly better compared to other immunosuppressive treatment and no immunosuppressive treatment, respectively, in terms of clinical remission ( 80 vs. 50 vs. $25 \%$ ) and renal survival (100, 80 , and $72 \%$ at 5 years).

Why are the results of the Caliskan group so different from those of the study of Rabasco et al. [8]? One possibility was a nonsignificant small admixture of DDD patients in the Turkish study ( 7 out of 60 , but 3 of them in the MMF limb. The Spanish study had 11 out of 23 patients who were tested positive for the $\mathrm{C} 3$ nephritic factor, but the risk of ESRD was lower in $\mathrm{C} 3 \mathrm{NeF}$-positive pa- tients when compared to $\mathrm{C} 3 \mathrm{NeF}$-negative patients. Turkish patients were older, but the mean age of patients treated with MMF was similar (32 vs. 35 years respectively); there was a major difference in the age of patients not treated with immunosuppression ( 29 vs. 40 years, respectively). Additionally, in both studies, there was a male preponderance and proteinuria was lower in patients on symptomatic treatment, although the difference was significant only in the Turkish study. There was a much shorter mean follow-up in the Turkish study (28 months) compared to the Spanish study (47 months). Serum creatinine at presentation seemed to be comparable. The magnitude of glomerulosclerosis above $50 \%$ was low in the Spanish study (5\%) and higher in the Turkish study $(18.5 \%)$ in the MMF-treated patients. Conversely, according to the Spanish study, moderate to severe interstitial fibrosis was present in $41 \%$ of MMF-treated patients but only in $11 \%$ in the Turkish study. The presence of crescents was not reported in the Spanish study but was reported in 55\% of Turkish patients treated with MMF.

Nonspecific antiproliferative strategies (cyclophosphamide or mycophenolate mofetil) are supposed to be less effective than specific complement-directed approaches (e.g., CFH replacement or targeting membrane attack). However, more precise information on the pathogenesis of C3 glomerulonephritis in a given individual as well as the presence of specific markers of complement activation of the different proteins pathway (e.g., C3c, C5, soluble membrane attack complex) and their inhibitors (e.g., $\mathrm{CFH}$ ), for example, anti-C5 antibody eculizumab will be needed to tailor therapy for treatment of this disease.

In conclusion, although there were some differences between the Spanish [8] and the Turkish study, the Turkish study does not confirm the putative efficacy of mycophenolate-based immunosuppressive treatment for C3 glomerulopathy. Definitely, in the diseases characterized by the activation (or lack of inhibition) of the alternative (and sometimes) terminal complement pathway, one would not expect antiproliferative treatment to be effective. To improve the poor renal outcome of patients with C3 glomerulopathy, we need to better understand the mechanism of complement activation in each individual patient with subsequent personalized treatment directed at the inhibition of the specific identified step with targeted treatment.

\section{Disclosure Statement}

The authors have no conflicts of interest to disclose.
Tesar/Hruskova 


\section{References}

1 Caliskan Y, Torun ES, Tiryaki TO, Oruc A, Ozluk Y, Akgul SU, Temurhan S, Ozrop N, Klicaslan I, Sever MS: Immunosuppressive treatment in C3 glomerulopathy: is it really effective? Am J Nephrol 2017;46:96-107.

2 Pickering MC, D'Agati VD, Nester CM, Smith RJ, Haas M, Appel GB, Alpers CE, Bajema IM, Bedrosian C, Braun M, Doyle M, Fakhouri F, Fervenza FC, Fogo AB, FrémeauxBacchi V, Gale DP, Goicoechea de Jorge E, Griffin G, Harris CL, Holers VM, Johnson S, Lavin PJ, Medjeral-Thomas N, Paul Morgan B, Nast CC, Noel LH, Peters DK, Rodríguez de Córdoba S, Servais A, Sethi S, Song WC, Tamburini P, Thurman JM, Zavros M, Cook HT: C3 glomerulopathy: consensus report. Kidney Int 2013;1079-1089.

3 Servais A, Noel LH, Rooumenina LT, et al: Acquired and genetic complement abnormal- ities play a critical role in dense deposit disease and other C3 glomerulopathies. Kidney Int 2012;82:454-464.

4 Servais A, Frémeaux-Bacchi V, Lequintrec M, et al: Primary glomerulonephritis with isolated C3 deposits: a new entity which shares common genetic risk factors with haemolytic uraemic syndrome. J Med Genet 2007;44: 193-199.

5 Smith RJ, Alexander J, Barlow PN, et al: New approaches to the treatment of dense deposit disease. J Am Soc Nephrol 2007; 18:24472456.

6 Medjeral-Thomas NR, O’Shaughnessy MM, O’Regan JA, Traynor C, Flanagan M, Wong L, Teoh CW, Awan A, Waldron M, Cairns T, O'Kelly P, Dorman AM, Pickering MC, Conlon PJ, Cook TH: C3 glomerulopathy: clinicopathologic features and predictors of outcome. Clin J Am Soc Nephrol 2014;9:4653.

7 McCaughan JA, O'Rourke DM, Courtney AE: Recurrent dense deposit disease after renal transplantation: an emerging role for complementary therapies. Am J Transplant 2012;12: 1046-1051.

8 Rabasco C, Cavero T, Román E, Rojas-Rivera J, Olea T, Espinosa M, Cabello V, FernándezJuarez G, González F, Ávila A, Baltar JM, Díaz M, Alegre R, Elías S, Antón M, Frutos MA, Pobes A, Blasco M, Martín F, Bernis C, Macías M, Barroso S, de Lorenzo A, Ariceta G, LópezMendoza M, Rivas B, López-Revuelta K, Campistol JM, Mendizábal S, de Córdoba SR, Praga M; Spanish Group for the Study of Glomerular Diseases (GLOSEN): Effectiveness of mycophenolate mofetil in C3 glomerulonephritis. Kidney Int 2015;88:1153-1160. 